\title{
HLA-DR and tuberculin tests in rheumatoid arthritis and tuberculosis
}

G M BAHR, ${ }^{1}$ M A SATTAR, ${ }^{1} \mathrm{~J}$ L STANFORD ${ }_{3}^{2}$ M A SHAABAN, B Al SHIMALI, ${ }^{3}$ Z SIDDIQUI, ${ }^{3}$ M GABRIEL, ${ }^{3}$ M Al SAFFAR, A SHAHIN, ${ }^{1}$ T D CHUGH, ${ }^{1}$ G A W ROOK, ${ }^{2}$ AND K BEHBEHANI

From the ${ }^{1}$ Departments of Microbiology and Medicine, Faculty of Medicine, Kuwait University, PO Box 24923, Kuwait; the ${ }^{2}$ School of Pathology, University College and Middlesex School of Medicine, London; and the ${ }^{3}$ Chest Diseases Hospital, Kuwait

SUMMARY Responses to four new tuberculins were found to be significantly reduced in 46 patients with rheumatoid arthritis in comparison with a control group of 79 . Except for tuberculin itself, the same was found in 111 patients with tuberculosis. In common with patients with tuberculosis and leprosy, those with rheumatoid arthritis did not respond to common mycobacterial (group i) antigen. Three DR haplotypes were found to have significant effects on skin test responsiveness of the rheumatoid patients but had little or no effect on that of the patients with tuberculosis and none on that of the healthy control group. Rheumatoid patients with the HLA-DR4 haplotype had significantly greater responses to all four reagents than did non-DR4 patients, but their responses to leprosin A and scrofulin remained significantly lower than those of the control group. Possession of HLA-DR3 haplotype was associated with skin test positivity approaching normal, but the sizes of responses were reduced. Possession of DR7 was associated with an unexpected reduction in skin test positivity, especially in the case of tuberculin. These results support the hypothesis that mycobacteria, or autoantigens cross reactive with mycobacteria, may be involved in the aetiology of rheumatoid arthritis. The results also show that the regulation and specificity of responsiveness to mycobacterial antigens are different in patients with rheumatoid arthritis with different HLA-DR haplotypes.

Recent evidence has reopened the question of a relation between mycobacteria, or autoantigens cross reactive with mycobacteria, and rheumatoid arthritis, perhaps similar to that observed in the rat adjuvant arthritis model. ${ }^{12}$ One report presented evidence for an unexpected association between HLA-DR4 and the size of tuberculin responses in patients with leprosy. ${ }^{3}$ As HLA-DR4 is known to be strongly associated with rheumatoid arthritis in Western populations, ${ }^{4}$ and as tuberculin test responsiveness is known to be decreased in rheumatoid arthritis, ${ }^{5}$ we investigated HLA type in relation to four mycobacterial skin tests in patients with this disease. In addition to the individual results of the skin tests, this set of four tests allows separation into three responder categories. ${ }^{6}$ Category 1 subjects

Accepted for publication 14 May 1988.

Correspondence to Dr G M Bahr, Department of Microbiology and Medicine, Faculty of Medicine, Kuwait University, PO Box 24923, Kuwait. produce positive reactions to all four reagents, in many cases owing to recognition of common mycobacterial (group i) antigens. ${ }^{7}$ Category 2 are nonresponders to all reagents, and category 3 respond to one or more reagents through recognition of their species specific (group iv) antigens.

To provide data generated simultaneously in individuals suffering from a known mycobacterial disease we studied a group of patients with tuberculosis and a healthy control group. Despite numerous studies no strong associations have been found between HLA-DR type and tuberculosis. Tuberculin responses are known to be variable in size, and the numbers of category 1 responders are known to be reduced in tuberculosis, ${ }^{8}$ but no attempt to find an association with DR type has been reported previously. Among healthy people lack of the determinant DR3 has been shown to be associated with failure to respond to any of a battery of four new tuberculins (category 2 , non-responders), ${ }^{9}$ but no association has been reported between sizes of 
responses and DR haplotype. Several studies have related the DR3 haplotype with tuberculoid leprosy and lack of it with lepromatous disease..$^{11}$ Homozygous DR3 macrophages have been shown to be poorly effective in presenting Mycobacterium leprae antigen to the $\mathrm{T}$ lymphocytes of $\mathrm{DR} 3$ homozygous or heterozygous patients with tuberculoid leprosy. ${ }^{12}$ Very recently a determinant on the 65 kilodalton protein common to all mycobacteria has been defined, to which all DR3 individuals respond ( $R$ de Vries, personal communication).

\section{Patients and methods}

PATIENTS WITH RHEUMATOID ARTHRITIS Forty six patients of Arab nationality (32 women, 14 men) attending the rheumatology clinic at Mubarak teaching hospital in Kuwait with established rheumatoid arthritis varying in duration from two to 18 years took part in the study. The severity of the disease varied from mild (23 patients) to moderate (19 patients), and only four had severe disease. Their ages ranged from 18 to 57 years with a mean of 37. Most patients were receiving treatment with gold (15 patients), penicillamine (17 patients), or chlorambucil (nine patients). None of them was known to have had mycobacterial diseases in the past.

PATIENTS WITH TUBERCULOSIS

One hundred and eleven patients with tuberculosis (92 men, 19 women) with ages ranging from 17 to 55 years (mean 32 ) took part in the study. Forty eight were Arabs and 63 were Indians or Pakistanis. All were inpatients of the Chest Diseases Hospital in Kuwait and had received one month's treatment for proved pulmonary tuberculosis at the time of skin testing.

HEALTHY CONTROL GROUP

The control group were 42 male and 37 female university and hospital staff whose ages ranged from
20 to 60 years (mean 35). Forty eight were Arabs and 31 Indians or Pakistanis.

\section{HLA TYPING}

Terasaki tissue typing plates coated with specific $\frac{\overline{\bar{S}}}{\bar{D}}$ antisera (One Lambda Incorporated, Los Angeles, $\stackrel{\mathbb{Q}}{\varrho}$ CA, USA) were used to determine the HLA-DR types of all subjects in this study. ${ }^{13} 14$

\section{SKIN TEST REAGENTS}

The skin test reagents were the new tuberculins: tuberculin, leprosin A, scrofulin, and vaccin, pre- $\frac{\varrho}{2}$ pared in London from Mycobacterium tuberculosis, $M$ leprae, $M$ scrofulaceum, and $M$ vaccae respectively. ${ }^{15}$ With the exception of leprosin $\mathrm{A}, \dot{\sigma}$ which was a new batch, the batches used were those which had been tested on the Spanish patients with leprosy. ${ }^{3}$ The same batches of reagents were used throughout the study. The doses used were stan- ָّ dard: $0.2 \mu \mathrm{g}$ of tuberculin and scrofulin, $1.0 \mu \mathrm{g}$ of leprosin $\mathrm{A}$, and $2.0 \mu \mathrm{g}$ of vaccin. Intradermal injections of $0.1 \mathrm{ml}$ were administered on the $\vec{\theta}$ forearms and diameters of induration read after $72 \%$ hours. Reactions of $2 \mathrm{~mm}$ or more were taken as positive.

\section{Results}

The skin test responses of the patients with arthritis were significantly fewer and, with the exception of $\bar{\partial}$ vaccin, smaller than those of the control group (Table 1). The patients with tuberculosis appeared similar to the control group in their response to tuberculin but had significantly fewer and smaller responses to the other three reagents.

Earlier studies have shown a change in category distribution and a loss of category 1 individuals among those with mycobacterial infections. ${ }^{6}$ This was supported by our present findings for patients $\mathrm{O}$ with tuberculosis, and was also seen among the

Table 1 Skin test parameters of healthy controls and of patients with rheumatoid arthritis or tuberculosis

\begin{tabular}{|c|c|c|c|c|}
\hline & \multicolumn{4}{|c|}{ Skin test reagent used } \\
\hline & Tuberculin & Leprosin A & Scrofulin & Vaccin \\
\hline $\begin{array}{l}\text { Rheumatoid arthritis } \\
\text { No, \% } \\
\text { Mean (SD) positive reaction size (mm) }\end{array}$ & $\begin{array}{l}25 / 46,54^{* *} \\
9 \cdot 5(6 \cdot 5)\end{array}$ & $\begin{array}{l}16 / 45,36^{* *} \\
6.6(3.4)\end{array}$ & $\begin{array}{l}13 / 46,28^{* *} \\
6 \cdot 5(2 \cdot 7)\end{array}$ & $\begin{array}{l}22 / 46,48^{*} \\
8 \cdot 8(3 \cdot 6)\end{array}$ \\
\hline $\begin{array}{l}\text { Healthy controls } \\
\text { No, \% } \\
\text { Mean (SD) positive reaction size (mm) } \\
\text { Tuberculosis }\end{array}$ & $\begin{array}{l}74 / 79,94 \\
13 \cdot 3(4 \cdot 9)\end{array}$ & $\begin{array}{l}55 / 78,71^{* *} \\
8 \cdot 8(2 \cdot 8)\end{array}$ & $\begin{array}{l}61 / 78,78^{* *} \\
8 \cdot 6(3 \cdot 1)\end{array}$ & $\begin{array}{l}59 / 78,76^{* *} \\
8 \cdot 0(2 \cdot 5)\end{array}$ \\
\hline $\begin{array}{l}\text { No, } \% \\
\text { Mean (SD) positive reaction size }(\mathrm{mm})\end{array}$ & $\begin{array}{l}96 / 111,86 \\
12 \cdot 4(3 \cdot 3)\end{array}$ & $\begin{array}{l}29 / 111,26 \\
6 \cdot 5(2 \cdot 9)\end{array}$ & $\begin{array}{l}27 / 111,24 \\
6 \cdot 3(2 \cdot 1)\end{array}$ & $\begin{array}{l}17 / 111,15 \\
5 \cdot 7(1 \cdot 5)\end{array}$ \\
\hline
\end{tabular}


patients with arthritis (Table 2 and Fig. 1). There was a compensatory increase in category 3 responders among the patients with tuberculosis, but among the patients with rheumatoid arthritis the compensatory increase was chiefly in category 2 , a finding reminiscent of leprosy.

There were no significant differences in HLA-DR type between the Arab and non-Arab controls or those with tuberculosis. Thus we have not divided our results according to nationality. Fourteen $(30 \%)$ of the 46 patients with arthritis and $18(16 \%)$ of the 111 patients with tuberculosis had the haplotype

Table 2 Categorisation of responders among the healthy controls and the patients with rheumatoid arthritis or tuberculosis

\begin{tabular}{|c|c|c|c|c|c|c|}
\hline & \multicolumn{2}{|c|}{ Category 1} & \multicolumn{2}{|c|}{ Category 2} & \multicolumn{2}{|c|}{ Category 3} \\
\hline & No & $\%$ & No & $\%$ & No & $\%$ \\
\hline Rheumatoid arthritis & s $8 / 46$ & $17^{*}$ & $19 / 46$ & $41^{*}$ & $19 / 46$ & 41 \\
\hline Healthy controls & $49 / 78$ & $63^{*}$ & $4 / 78$ & 5 & $25 / 78$ & $32^{*}$ \\
\hline Tuberculosis & $3 / 111$ & 3 & $10 / 111$ & 9 & $98 / 111$ & 88 \\
\hline
\end{tabular}

${ }^{*}$ Indicates $\mathrm{p}<0 \cdot 00001, \chi^{2}$ test after Yates's correction.
HLA-DR4 $(\mathrm{p}<0.02)$, but neither differed significantly from the control group $(20 / 79(25 \%))$. None of the other HLA types showed any disease association. In the control group HLA-DR haplotype did not effect responses to any of the skin test reagents. Table 3 shows that this was far from the case in the patients with rheumatoid arthritis.

Although possession of the haplotype DR4 made no difference to skin test responsiveness of patients with tuberculosis, it had a marked effect among the patients with arthritis. In comparison with the control group DR4 rheumatoid patients had similar responses to tuberculin and vaccin, but significantly reduced responses to leprosin $A$ and scrofulin $(\mathrm{p}<0.001$ and $\mathrm{p}<0.003$ respectively).

HLA-DR3 influenced responsiveness to skin tests in both patient groups (Table 3). Among the rheumatoid patients possession of haplotype DR3 was associated with greater positivity to tuberculin, leprosin $A$, and vaccin, which reached significance in the case of leprosin $A(p<0 \cdot 001)$, although the sizes of positive responses to tuberculin and leprosin A remained significantly smaller than in the control group ( $p<0.03$ in both cases). Among patients with tuberculosis DR3 did not influence responsiveness
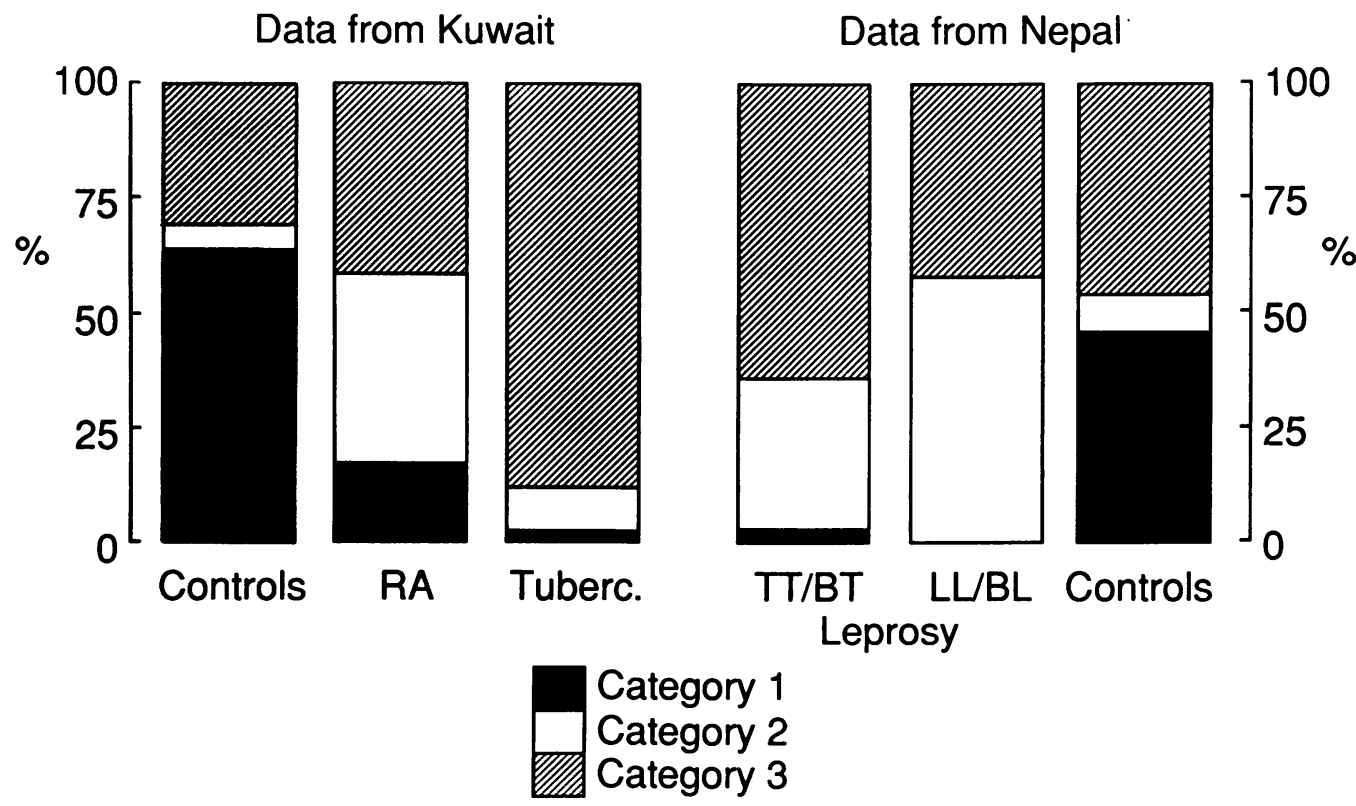

Fig. 1 Distribution of skin test responder categories in patients with rheumatoid arthritis, tuberculosis, and leprosy and comparison with their respective controls. (The data from Nepal were available from a previous study. ${ }^{19}$ ) $R A=$ patients with rheumatoid arthritis; Tuberc. = patients with tuberculosis; TT/BT=combined results for patients with polar tuberculoid and borderline tuberculoid leprosy; $L L / B L=$ combined results for patients with polar lepromatous and borderline lepromatous leprosy. 
Table 3 Skin test results divided according to HLA-DR type $\dagger$

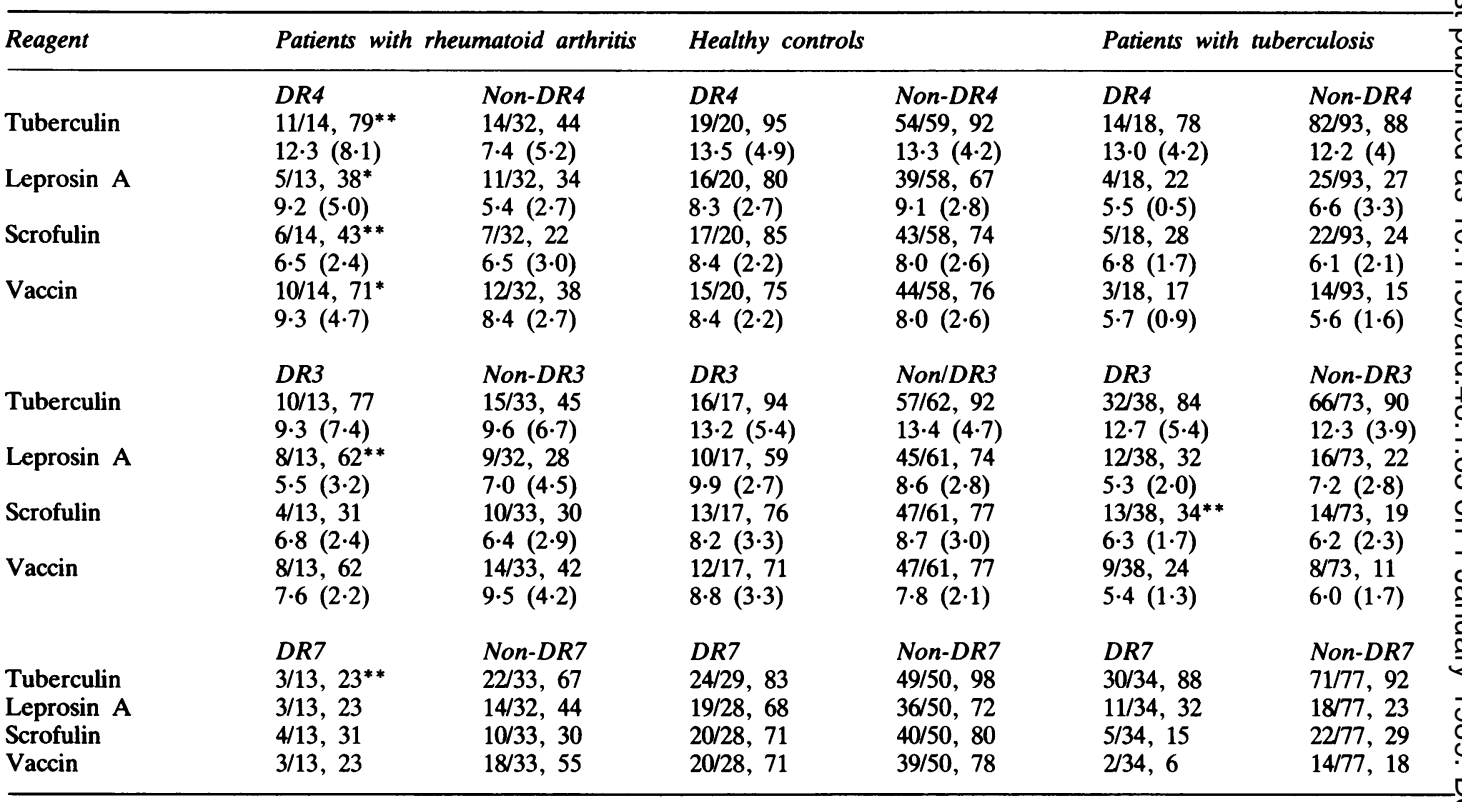

${ }^{*}$ Indicates $\mathrm{p}<0.01 ;{ }^{* *} \mathrm{p}<0 \cdot 001$, Mann-Whitney test.

The statistical results have not been corrected for the number of comparisons made in the table because the results obtained are those that were specifically sought except in the case of DR7 which were unexpected findings.

$\dagger$ The figures show number, percentage and, below, the mean (SD) positive reaction size in millimetres. There were no significanto differences in response sizes in the DR7 comparisons, and therefore they are not shown.

to tuberculin, but increased responsiveness to the other three reagents; significantly so in the case of scrofulin $(\mathrm{p}<0.001)$.

The only other haplotype found to influence skin test results was DR7, possession of which was associated with decreased responsiveness to tuberculin in rheumatoid patients, but had no effect among patients with tuberculosis or healthy controls (Table 3).

When the DR types were segregated according to responder category it was found that there was a significant association between DR7 haplotype and skin test non-responsiveness among patients with arthritis, which was not seen among patients with tuberculosis or controls.

\section{Discussion}

Our results showed that the incidence of HLA-DR4 was raised significantly among the patients with rheumatoid arthritis we studied in comparison with patients with tuberculosis but not in comparison with the control group. This is not surprising as the work associating this haplotype with the disease was carried out in a quite different population. We also showed that responsiveness to mycobacterial skin test reagents is strikingly reduced in patients with arthritis in comparison with a control group (Table 3 1) and is associated with DR haplotype. The differences found suggest that patients with arthritis are subject to some form of immune regulation of $\circ$ their responses to mycobacteria. The similarity between these patients and the patients with tuber- $\frac{D}{0}$ culosis (both DR4 and non-DR4) in their low responses to leprosin $A$ and scrofulin is striking and $N$ suggests similar regulatory mechanisms may control $N$ the responses to these reagents in both diseases. The $N$ almost normal responses to tuberculin and vaccin $\omega$ and the low responses to leprosin A and scrofulin among DR4 rheumatoid patients show that there is specificity in their responsiveness, and that they are not non-specific high responders.

The increased positivity to skin tests seen among patients with rheumatoid arthritis and tuberculosis with the haplotype DR3 suggests that they may? retain a degree of responsiveness to common mycobacterial antigen, possibly to the 65 kilodaltone protein ( $\mathrm{R}$ de Vries, personal communication). $\delta$ 
Thus the regulation of skin test responses to mycobacterial antigens in DR3 and DR4 rheumatoid patients is almost certainly different.

The discovery of an association between DR7 low tuberculin responses and category 2 non-responders to mycobacterial skin tests in patients with arthritis was a new and unexpected finding in our study. This was especially interesting as it has recently been shown that DR2, DR6, and DR7 are reduced in rheumatoid disease, at least in an American series. ${ }^{16}$ We looked, therefore, at the skin test responses of the 13 DR2 patients, but they did not differ significantly from the whole group. Only two rheumatoid patients in our study had haplotype DR6.

We have recently demonstrated a low, class specific antibody response to mycobacterial antigens in DR7 and DR2 but not DR4 patients with rheumatoid arthritis, which was not seen in patients with tuberculosis. ${ }^{17}$ When this is considered in combination with the present data it is difficult to propose an aetiology for rheumatoid disease that does not involve mycobacteria in some way.

It has previously been reported that patients with mycobacterial infections do not give positive skin test responses to common mycobacterial (group i) antigens, ${ }^{68}$ probably because of a regulatory mechanism (see Fig. 1). We have now found this amongst rheumatoid patients, and it may also explain the reduced reponses to leprosin $A$ and scrofulin in those with DR4 if their reactions to tuberculin and vaccin are to species specific (group iv) antigens in these preparations.

Most of our control group, about half of the rheumatoid patients, and $20 \%$ of the patients with tuberculosis had the scars of BCG vaccination, but we could not find an association between skin test responses and the presence of BCG scars in our patient groups. Similarly, we were unable to associate any of the treatments for arthritis with any particular pattern of results.

In conclusion, our observations reinforce the suggestion of some relation between mycobacteria and rheumatoid arthritis. The proposal that the relation is purely due to the propensity for DR4 individuals to respond strongly to mycobacterial antigens is an insufficient explanation. ${ }^{18}$ The marked reduction in numbers of rheumatoid patients responding to common mycobacterial antigen is similar to that seen in our patients with tuberculosis, and previously reported in leprosy. ${ }^{619}$ The expansion of category 2 non-responders seen in arthritic patients is also similar to that previously described in leprosy. ${ }^{6}$ This similarity between rheumatoid arthritis and leprosy is seen additionally in the associations between large sized responses to tuberculin and DR4 haplotype, ${ }^{3}$ and between small sized reactions to leprosin A and DR3 haplotype reported in tuberculoid leprosy. ${ }^{10-12} \mathrm{~A}$ syndrome closely resembling rheumatoid arthritis occurs in some forms of reaction in multibacillary leprosy, ${ }^{20-22}$ and is associated with development of rheumatoid factor. If our results really mean that the immunopathology of rheumatoid arthritis is similar to that of mycobacterial infections then contact with environmental mycobacteria (perhaps by mouth) may regulate a tissue damaging immune response directed at antigenic determinants which joints are known to share with mycobacteria.

We would like to thank $\mathbf{R}$ George and I Abdul Latif for their technical assistance, B Xavier for secretarial help, and Dr R J W Rees of the National Institute for Medical Research, London for provision of the leprosin A. This work was supported by Kuwait University Research Grants MI 034, MI 006, MI 008 and by grant No 84-07-01 from the Kuwait Foundation for the Advancement of Science. The production of antigens in the United Kingdom was supported by Lepra.

\section{References}

1 Holoshitz J, Naparstek Y, Ben-Nun A, Cohen I R. Lines of T lymphocytes induce or vaccinate against autoimmune arthritis. Science 1983; 219: 56-8.

2 Holoshitz J, Matitiau A, Cohen I R. Arthritis induced in rats by cloned $\mathrm{T}$ lymphocytes responsive to mycobacteria but not to collagen type II. J Clin Invest 1984; 73: 211-5.

3 Ottenhoff $\mathrm{T} \mathrm{H}$, Torres $\mathrm{P}$, Terencio J, et al. Evidence for an HLA-DR4 associated immune response gene for $M$. tuberculosis. A clue to the pathogenesis of rheumatoid arthritis. Lancet 1986; ii: $310-3$.

4 Panayi G S, Wooley P H, Batchelor J R. Genetic basis of rheumatoid arthritis: HLA antigens, disease manifestations and toxic reactions to drugs. $\mathrm{Br}$ Med $J$ 1978; ii: $1326-8$.

5 Waxman J, Lockshin M D, Schnapp J J, Doneson I N. Cellular immunity in rheumatic diseases. 1. Rheumatoid arthritis. Arthritis Rheum 1973; 16: 499-506.

6 Stanford J L, Nye P M, Rook G A W, Samuel N O, Fairbank A. A preliminary investigation of the responsiveness or otherwise of patients and staff of a leprosy hospital to groups of shared or species specific antigens of mycobacteria. Lepr Rev 1981; 52: 321-7.

7 Stanford J L, Grange J M. The meaning and structure of species as applied to mycobacteria. Tubercle 1974; 55: 143-52.

8 Stanford J L. Immunologically important constituents of mycobacteria: antigens. In Ratledge $\mathrm{C}$, Stanford $\mathrm{J} \mathrm{L}$, eds. The biology of the mycobacteria. Vol 2. London: Academic Press, 1983: 85-128.

9 Van Eden W, De Vries R R, Stanford J L, Rook G A W. HLADR3 associated genetic control of response to multiple skin tests with new tuberculins. Clin Exp Immunol 1983; 52: 287-92.

10 Van Eden W, De Vries R R P, d'Amaro J, Schreuder I, Leiker D L, Van Rood J J. HLA-DR associated genetic control of the type of leprosy in a population from Surinam. Hum Immunol 1982; 4: 343-50.

11 Van Eden W, Gonzales N M, De Vries R R P, Convit J, Van Rood J J. HLA-segregation in multi-case leprosy families indicating HLA-linked control of predisposition to lepromatous leprosy. J Infect Dis 1985; 151: 9-14.

12 Van Eden W, Elferink B G, De Vries R R P, Leiker D L, Van Rood J J. Low T-lymphocyte responsiveness to Mycobacterium leprae antigens in association with HLA-DR3. Clin Exp Immunol 1983; 55: 140-8. 
13 Van Rood J J, Van Leeuwen A, Ploem J S. Simultaneous detection of two cell populations by two-colour fluorescence and application to the recognition of B-cell determinants. Nature 1976; 262: 795-7.

14 Al Din A S N, Al Saffar M, Siboo R, Behbehani K. Association between HLA-D region epitopes and multiple sclerosis in Arabs. Tissue Antigens 1986; 27: 196-200.

15 Shield M J, Stanford J L, Paul R C, Carswell J W. Multiple skin testing of tuberculosis patients with a range of new tuberculins, and a comparison with leprosy and $\mathrm{M}$. ulcerans infection. $\mathrm{J} \mathrm{Hyg}$ (Lond) 1977; 78: 331-48.

16 Stastny P, Ball E J, Khan M A, Olsen N J, Pincus T, Gao X. HLA-DR4 and other genetic markers in rheumatoid arthritis. $\mathrm{Br} J$ Rheumatol (in press).

17 Bahr G M, Rook G A W, Shahin A, Stanford J L, Sattar M A, Behbehani K. HLA-DR associated isotype-specific regulation of antibody levels to mycobacteria in rheumatoid arthritis. Clin Exp Immunol 1988; 72: 26-31.

18 Panayi G S. Rheumatoid arthritis and tuberculosis. Lancet 1986; ii: 816 .

19 Morton A, Nye P, Rook G A W, Samuel N, Stanford J L. A further investigation of skin-test responsiveness and suppression in leprosy patients and healthy school children in Nepal. Lepr $\operatorname{Rev} 1$ 1984; 55: 273-81.

20 Ramu G, Ramunajam K. Reactive states in lepromatous $\mathcal{O}$ leprosy. Leprosy in India 1964; 36: 3-19.

21 Ramu G, Balakrishnan S. Arthritis in lepromatous leprosy. Leprosy in India 1968; 40: 1-8.

22 Atkin S L, Welbury R R, Stanfield E, Beavis D, Iwais B, Dick W C. Clinical and laboratory studies of inflammatory polyarthritis in patients with leprosy in Papua New Guinea. Ann Rheum Dis 1987; 46: 688-90. 